\title{
Emotion perception, non-social cognition and symptoms as predictors of theory of mind in schizophrenia
}

Anja Vaskinn ${ }^{\mathrm{a}, \mathrm{b}^{*}}$, Stein Andersson ${ }^{\mathrm{c}}$, Tiril $\emptyset_{\text {stefjells }}^{\mathrm{a}, \mathrm{d}}$, Ole A. Andreassen ${ }^{\mathrm{a}, \mathrm{b}}$, Kjetil Sundet $^{\mathrm{a}, \mathrm{c}}$

a) NORMENT KG Jebsen Centre for Psychosis Research, Oslo University Hospital, P.O.Box 4959 Nydalen, 0424 Oslo, Norway

b) Institute of Clinical Medicine, University of Oslo, P.O.Box 1171 Blindern, 0318 Oslo, Norway

c) Department of Psychology, University of Oslo, P.O.Box 1094 Blindern, 0317 Oslo, Norway

d) Department for Specialized Inpatient Treatment, Division of Mental Health and Addiction, Akershus University Hospital, 1478 Lørenskog, Norway

* Corresponding author:

Anja Vaskinn

NORMENT KG Jebsen Centre for Psychosis Research

Oslo University Hospital

P.O.Box 4959 Nydalen

0424 Oslo, Norway

anja.vaskinn@medisin.uio.no

Phone: + 4723027331

Fax: + 4723027333 


\begin{abstract}
Background: Theory of mind (ToM) can be divided into cognitive and affective ToM, and a distinction can be made between overmentalizing and undermentalizing errors. Research has shown that ToM in schizophrenia is associated with non-social and social cognition, and with clinical symptoms. In this study, we investigate cognitive and clinical predictors of different ToM processes.
\end{abstract}

Methods: Ninety-one individuals with schizophrenia participated. ToM was measured with the Movie for Assessment of Social Cognition (MASC) yielding six scores (total ToM, cognitive ToM, affective ToM, overmentalizing errors, undermentalizing errors and no mentalizing errors). Neurocognition was indexed by a composite score based on the nonsocial cognitive tests in the MATRICS Consensus Cognitive Battery (MCCB). Emotion perception was measured with Emotion in Biological Motion (EmoBio), a point-light walker task. Clinical symptoms were assessed with the Positive and Negative Syndrome Scale (PANSS). Seventy-one healthy control (HC) participants completed the MASC.

Results: Individuals with schizophrenia showed large impairments compared to $\mathrm{HC}$ for all MASC scores, except overmentalizing errors. Hierarchical regression analyses with the six different MASC scores as dependent variables revealed that MCCB was a significant predictor of all MASC scores, explaining 8-18\% of the variance. EmoBio increased the explained variance significantly, to $17-28 \%$, except for overmentalizing errors. PANSS excited symptoms increased explained variance for total ToM, affective ToM and no mentalizing errors.

Discussion: Both social and non-social cognition were significant predictors of ToM. Overmentalizing was only predicted by non-social cognition. Excited symptoms contributed to overall and affective ToM, and to no mentalizing errors.

Keywords: social cognition, emotion perception, theory of mind, mentalizing

\title{
Highlights:
}

- Schizophrenia is characterized by a global impairment in ToM which is present for both cognitive and affective dimensions

- The impairment seems to be based largely on insufficient mentalizing as number of overmentalizing errors did not differ significantly compared to healthy controls

- Although ToM is a complex concept, different types of ToM are dependent upon the same processes; i.e. non-social cognition and low-level social cognition/emotion perception 


\section{Introduction}

The ability to infer the intentions, dispositions and beliefs of others has been labeled theory of mind (ToM) [1]. The term was introduced by Premack \& Woodruff [2], and ToM is today considered part of the broader concept of social cognition, often defined as "the mental operations that underlie social interactions, including perceiving, interpreting and generating responses to the intensions, dispositions and behaviors of others" [3]. In addition to ToM, social cognition includes emotion perception, social perception and attributional style [1]. Individuals with schizophrenia (SZ) are impaired in all domains of social cognition [4], and social cognition is an important determinant of real-world outcomes for persons with SZ [1]. Social cognition mediates between non-social cognition and outcome [5], and ToM seems to be an especially strong predictor of functioning [6].

ToM is a complex cognitive function, not fully developed until adulthood [7]. It can be conceptualized in different ways. For example, ToM can be understood according to a) level of processing [8], b) hot versus cold cognition [9], or c) mentalizing style [10]. According to the first approach, social cognition might best be conceptualized based on level of processing rather than domain of social information [11]. In such a perspective, emotion perception has been considered a lower-level and ToM a higher-level cognitive process [8, 12]. From this follows that (low-level) emotion perception should predict (high-level) ToM [12]. Related, it seems logical that higher level social cognition, such as ToM, is dependent upon non-social cognition. There is empirical evidence for an association between ToM and non-social cognition, such as speed of processing, visual and verbal learning and memory, executive function [13], and IQ [14, 15]. However, other studies do indicate that ToM and non-social cognition can dissociate $[16,17]$

Another approach to social cognition and ToM is the differentiation between "hot" and "cold" [18] or affective and cognitive dimensions of ToM [9]. Whereas cognitive ToM requires cognitive understanding of the difference between the knowledge of the speaker and of the listener (i.e. knowledge about beliefs, traditionally assessed with false belief tasks), affective ToM involves an empathic appreciation of another person's emotional state (knowledge about emotions, traditionally assessed with irony or faux pas tasks) [9]. Attribution of another person's internal state is involved in both cognitive and affective ToM. The difference is the type of internal state that is attributed. Attributions of thoughts, knowledge or action plans make up cognitive ToM, whereas attributions of emotional states like anger or guilt is referred 
to as affective ToM [19]. Based on theories which separate between cognitive and affective ToM dimensions, one could hypothesize that emotion perception would predict affective ToM, but not cognitive ToM. Instead, non-social cognition would be an expected determinant of cognitive ToM. Differential associations between the characteristic symptoms of SZ and cognitive and affective ToM lend some support to the validity of this distinction. Significant relationships have been reported between cognitive ToM and positive symptoms $[9,19]$, and between affective ToM and negative symptoms [9]. In one of the studies [19], the association with negative symptoms was significant for both cognitive and affective ToM, but not after controlling for age.

The third approach to ToM is of relevance for SZ symptomatology. This approach differentiates between mentalizing styles, i.e. the processes underlying incorrect ToM responses. Incorrect ToM performance can be due to overmentalizing, undermentalizing or a lack of mentalizing. To overmentalize is to excessively attribute intentions or self-referential meaning to others. Undermentalizing refers to a diminution in the ability to understand and attribute mental state, whereas a lack of mentalizing refers to a thinking style where no attributions are made to the mental state of another. A focus on mentalizing styles may help explain some of the inconsistencies found in the literature regarding associations between clinical symptoms and ToM [14]. Whereas some studies did not reveal significant associations between ToM and positive and negative symptoms [6, 20], others have found associations between ToM and disorganized, negative and positive symptoms [21, 22]. A meta-analysis [23] found medium effect size relationships with disorganization and negative symptoms, but not with reality distortion. The fact that ToM is a broad construct, consisting of different components could be one explanation for the inconsistent results. For example, different mentalizing styles appear to have different predictors. Whereas overmentalizing has been found to be associated with positive symptoms [15, 19] and attention/working memory [24], lack of mentalizing has been reported to be associated with negative symptoms [19], and undermentalizing (including lack of mentalizing) with emotion perception and verbal memory [24].

A related term is metacognition, or the ability to reflect on thoughts and feelings and connect these events into detailed representations of oneself or others [25]. One of four metacognitive domains, the ability to understand the mind of others, clearly overlaps with ToM. Indeed, metacognition shows many of the same characteristics as social cognition: it is related to cognition $[26,27]$ and negative symptoms $[27,28]$ and mediates between non-social 
cognition and functional outcome [29]. But whereas metacognition involves a synthesis of discrete processes into complex representations of others or oneself [30], social cognition make up some of these discrete processes. If we are to understand the details of how metacognition or social cognition relates to functional difficulties in schizophrenia, we need to disentangle how different discrete processes relate to each other. This can subsequently inform us of relevant treatment targets.

Knowledge of predictors of ToM is important when tailoring clinical interventions in SZ. Whereas social cognition training can lead to significant improvements in real-world outcome $[31,32]$, effects are small. One among several possible explanations is that social cognition training programs do not target the most relevant functions. If we can unveil the predictors of impaired ToM, an important determinant of functional outcome, we may also unveil important treatment targets. Currently, it remains unknown whether interventions are more likely to bring positive effects if they target low versus high-level processes, or whether a focus on non-social cognition is likely to benefit those receiving the training. This is the first study to concurrently investigate the impact of clinical and cognitive predictors of ToM, an important determinant of functional outcome, using well-validated measures and a large representative sample of individuals with SZ.

Our first research aim is to examine whether persons with SZ perform worse than healthy control participants (HC) on an ecological valid ToM measure, the Movie for the Assessment of Social Cognition (MASC) [33]. We hypothesize reduced performance for the total score, as well as for both cognitive and affective ToM. We also expect that the SZ group will commit significantly more errors, regardless of type, than the HC group.

Our second research aim is to examine predictors of ToM performance in participants with SZ. We hypothesize that emotion perception and non-social cognition will contribute significantly to overall ToM. Because of the mixed findings in the literature, we make no hypotheses concerning the predictive power of clinical symptoms on overall ToM. Further, we hypothesize that emotion perception and negative symptoms will predict affective ToM, and that non-social cognition and positive symptoms will predict cognitive ToM. Finally, based on previous findings, we expect overmentalizing errors to be predicted by non-social cognition and positive symptoms; undermentalizing errors to be predicted by non-social cognition, emotion perception, as well as by disorganized and negative symptoms; and no 
mentalizing errors to be predicted by non-social cognition, emotion perception and negative symptoms.

\section{Method}

\subsection{Participants}

Ninety-one individuals with a diagnosis of schizophrenia $(n=69)$ or schizoaffective disorder $(n=22)$ were recruited from hospitals in the greater Oslo area. All were participants in the Thematically Organized Psychosis (TOP) Study at the Norwegian Centre for Mental Disorders Research (NORMENT) at Oslo University Hospital. Inclusion criteria were Norwegian as mother tongue or all compulsory schooling conducted in Norway and age between 18-55 years. Exclusion criteria were IQ $\leq 70$ assessed with the 2-subtest Wechsler Abbreviated Scale of Intelligence (WASI) [34]; neurological disease, or head trauma causing hospitalization. Healthy control participants (HC: $\mathrm{n}=71)$ from the same geographical area were randomly selected from national statistical records and invited by letter. Before inclusion, $\mathrm{HC}$ were screened for symptoms of severe mental illness and excluded from the study if mental, neurological or somatic disorder was confirmed or suspected on the Primary Care Evaluation of Mental Disorders interview (PRIME-MD) [35]. Demographic and clinical information is displayed in Table 1. Participants in both groups were in their late 20s and slightly biased toward the male sex. The HC group was, as expected, characterized by longer education and higher IQ. For SZ, Global Assessment of Functioning (GAF) [36] scores indicate symptoms and functioning typical of a mostly outpatient population.

Insert Table 1 about here

\subsection{Measures}

\subsubsection{Clinical measures}

Diagnoses were based on the Diagnostic and Statistical Manual for Mental Disorders [37] using the Structured Clinical Interview for DSM-IV (SCID-I) [38]. Clinical data was collected by trained clinical psychologists or medical doctors and included the Positive and Negative Syndrome Scale (PANSS) [39] and the split GAF [36]. Symptoms were assessed using Wallwork's five-factor PANSS model [40] as several studies [41-43] have found it to provide 
the most optimal fit. It consists of the following factors (items): positive (P1, P3, P5, G9), negative (N1, N2, N3, N4, N6, G7) disorganized (P2, N5, G11), exited (P4, P7, G8, G14), and depressive (G2, G3, G6).

\subsubsection{Social cognitive measures}

ToM was assessed with the video-based MASC test [33]. The video shows a Saturday night dinner situation where two women and two men meet. The video is paused 45 times. Each time the participant is presented with a multiple choice question concerning the given character's mental state. There are four response options: (i) correct (MASCtom), (ii) overmentalizing (MASCexc), (iii) undermentalizing (MASCless), and (iv) no mentalizing (MASCno). Questions can also be divided into cognitive (MASCcog) and affective (MASCaff) ToM defined by which dimension they address. As there is no consensus in the MASC literature on the categorization of items [19, 44, 45], we conducted the following categorization: an item was allocated to the mental state dimension the question referred to (emotions: what does $X$ feel?; thoughts: what does $Y$ think?; intentions: why does $Z$ say this?). There were two exceptions to this general rule. For one item (\# 35), although a correct answer requires mentalizing, the mental state modality in the question (intention) is not among the response options (all describe factual circumstances - not thoughts, emotions or intentions). Therefore, this item did not receive a mental state modality. For another item (\# 42), the question referred to intentions, but the correct response describes a thought. Consequently, this item received the mental state modality of thought. Nine items were categorized as thoughts (item 6, 15, 18, 24, 28, 29, 36, 39, 42) and 17 as intentions (items 2, 3, 5, 7, 11, 16, $17,19,21,23,27,31,32,37,41,43,44)$, together making up 26 items for cognitive ToM (MASCcog); 18 items referred to emotions, or affective ToM (MASCaff - items 1, 4, 8, 9, 10, $12,13,14,20,22,25,26,30,33,34,38,40,45)$.

Emotion perception was assessed with the Emotion in Biological Motion test (EmoBio) [40], which consists of full-figure point-light displays (PLDs) of a person walking in a manner indicative of an emotion. It is a test of the ability to perceive emotions from body movement. We used the stimuli as adapted by Couture et al. [47], but with Norwegian norms [48]. It is comprised of 22 short clips of PLD walkers displaying the following emotions: angry, happy, sad, fearful, or no emotion. 


\subsubsection{Cognitive measures}

Non-social cognition was assessed with the Matrics Consensus Cognitive Battery (MCCB) [49], excluding the social cognition subtest. We used a composite score for the six non-social cognitive domains in the MCCB (speed of processing, attention/vigilance, working memory, verbal learning, visual learning, reasoning and problem solving).

\subsection{Statistical analyses}

Analyses were performed using The Statistical Package for the Social Sciences (IBM SPSS Statistics for Windows, Version 24.0, IBM Corp, Armonk, NY). Performance of the SZ and HC groups was compared with univariate analysis of variance (ANOVA) or chi-square analysis. Six separate hierarchical linear regression analyses were performed with each of the ToM variables (MASCtom, MASCexc, MASCless, MASCno, MASCcog, MASCaff) entered as dependent variable. Non-social cognition (MCCB) was entered in Step 1, emotion perception (EmoBio) in Step 2, and the five PANSS symptom factors in Step 3. These analyses were only conducted for the SZ group. The empirical and theoretical support for an association between cognition and ToM is more substantial than for clinical symptoms and ToM. We therefore used the SPSS “enter" command for variables in Step 1 and 2, and the "stepwise" command for the PANSS variables in Step 3. The fact that few relevant studies have utilized the five-factor PANSS model is another reason for choosing the "stepwise" command in Step 3.

Missing data was treated in the following way. One participant with SZ had one missing item on the EmoBio test. The most commonly given response in the SZ group (“anger”) was entered for this item for this participant. Five other participants with SZ did not undergo assessments with the EmoBio test and were excluded from the regression analyses. Six participants had missing data for one (four participants with SZ) or three (one participant with SZ, one HC) MCCB subtests. The mean SZ or HC group T-score, respectively, for the subtests in question was entered before a composite MCCB T-score was calculated and used for the statistical analyses.

Insert Table 2 about here 


\section{Results}

Scores on PANSS, EmoBio, MCCB \& MASC are shown in Table 2. ANOVAs yielded significant group differences for all ToM variables, in the expected direction, except for MASCexc. Similarly, the SZ group was impaired in non-social cognition, and their EmoBio score indicated impaired emotion perception based on normative data [48].

Insert Table 3 about here

The regression analyses for the six ToM measures are presented in Table 3. Non-social cognition (MCCB) was a significant predictor of all ToM outcome measures (MASC variables). Adding emotion perception (EmoBio) to the prediction increased the explained variance for all types of ToM, with the exception of overmentalizing errors. The inclusion of symptom dimensions (PANSS scores) in the third step of the regression analyses increased the explained variance for MASCtot, MASCaff, and MASCno, via PANSS excited symptoms. Our models explained from $11 \%$ (overmentalizing errors) to $32 \%$ (overall MASC score) of the variance in ToM performance. See Table 3 for details of the models.

\section{Discussion}

In this study, we examined different types of ToM components in individuals with SZ. As expected, persons with SZ had a ToM deficit compared to HC. This was the case for overall ToM performance level, as well as for cognitive and affective ToM. Effect sizes were large (Cohen's $d=0.79-1.06$ ). Our SZ group made more errors than HCs, significantly so for undermentalizing and no mentalizing types (large effect sizes/Cohen's $d=0.98-1.08$ ), but not for overmentalizing errors (small effect size/Cohen's $d=0.25$ ).

Our results corroborate previous research that has identified substantial impairments in ToM in this population [14]. We extend previous research by showing that the ToM impairment is of a global nature, in the sense that it is present for both cognitive and affective ToM. Further, the impairment seems to be based largely on insufficient mentalizing as number of overmentalizing errors did not differ significantly compared to HC. This is in line with a 
former study that used the same ToM test [24]. Similarly, in another study that compared MASC performance in individuals with SZ with that of $\mathrm{HC}$, significant case-control differences in overmentalizing errors vanished after controlling for verbal memory [19]. Such results contradict theories that postulate that SZ, characterized by delusions and paranoid ideation, would be associated with overmentalizing, in contrast to autism, characterized by lack of social reciprocity and deficits in non-verbal communication, which would be associated with undermentalizing $[10,50]$. One explanation for such findings in our sample is that it has a low psychotic symptom load. Another is that undermentalizing occurs naturally in SZ. SZ symptomatology extends beyond positive psychotic symptoms, and autism and SZ can co-occur in an individual, both at diagnostic and symptom levels [51, 52]. Chisholm et al. [51] reported in their review that an average of $24.1 \%$ of individuals with SZ also had autism. With this in mind, the presence of reduced mentalizing in SZ is not surprising. We also note that although we identified substantial impairments in ToM, the impairments for non-social cognition (MCCB composite score) were even larger (Cohen's $d=1.46$ ). This confirms that $\mathrm{SZ}$ is a disorder of cognitive aberrations [53].

Our analyses of predictors of ToM in SZ showed that non-social cognition and low-level emotion perception are of importance to complex social cognition. Non-social cognition was a significant predictor of all assessed ToM processes, explaining from 8 to $18 \%$ of the variance in the outcome variable. The addition of emotion perception in the model increased the explained variance to 17 to $28 \%$. In fact, emotion perception was a stronger predictor than non-social cognition when both were included in the model. The exception was for overmentalizing errors, which, as hypothesized, were found not to be dependent upon emotion perception. The expansion of the model to include SZ symptomatology increased explained variance significantly for overall ToM (32\%), affective ToM (27 \%), and for no mentalizing errors $(21 \%)$. The increased predictive validity of the model relied on the excited symptom factor.

The findings are largely in line with our hypotheses when it comes to the predictive power of non-social cognition and emotion perception to ToM. We found both to be of importance for the ability to make inferences about another person's mental state. This supports the suggested distinction between low and high levels of (social) cognitive processes $[8,12]$. We could, however, not confirm that emotion perception is more important for affective than for cognitive mental state reasoning. Similarly, non-social cognition influenced both cognitive 
and affective ToM. Therefore, our study does not support theories that differentiate between hot and cold ToM dimensions $[9,18]$.

Our findings concerning the effect of SZ symptomatology did not support our hypotheses and stand in contrast to previous research, including our own. In a smaller, but partly overlapping sample, we reported a significant association between overmentalizing errors and positive symptoms [15]. In the present analyses, none of the ToM components were found to be dependent upon the hypothesized PANSS symptoms, i.e. positive, negative, or disorganized symptoms. We suspect that different findings can emerge in studies of more symptomatic samples. A literature review [14] concluded that ToM impairments were larger in remitted compared to non-remitted samples. This could also mean that similar associations with clinical symptoms are not expected in remitted and non-remitted study samples. Indeed, some of the inconsistencies in the previous research could be due to heterogeneity of study populations. They could also be due to the use of different methodology to assess ToM, or even to assess clinical symptoms. We found that PANSS excited symptoms increased explained variance for three of our ToM variables. This was not expected based on previous findings, but perhaps not surprising as previous studies have used other PANSS models. The PANSS items in the excited factor concern hyperactivity (P4), hostility (P7), uncooperativeness (G8), and poor impulse control (G14). These symptoms not only reduce the likelihood of partaking in mutually satisfying, social exchanges, but also of thinking thoroughly about the perspective of others. From a clinical perspective it may therefore not be entirely unexpected that these symptoms could contribute to ToM performance.

\subsection{Limitations}

The present study has some limitations. Although our neuropsychological test battery (MCCB) was developed for the (repeated) assessment of cognition in SZ, it has been criticized for its somewhat limited ability to adequately index executive function [54]. A thorough investigation of the relationship between ToM and executive function requires a different neuropsychological test battery. Also, as evidenced by the modest degree of explained variance, it is clear that ToM must be related to something that we have not investigated.

\subsection{Conclusion/Implications}


In summary, our results indicate that different ToM components have more or less the same predictors, at least when analyses contain the predictors that were possible for us to include in this study.

This study suggests that although ToM is a complex concept, different types of ToM are dependent upon the same processes; i.e. non-social cognition and low-level social cognition/emotion perception. It also suggests that social cognition training could benefit from the inclusion of both non-social cognition and emotion perception as combined treatment targets in order to improve ToM functioning. Our findings are also of relevance for psychological treatments besides social cognition training. A number of psychotherapies focus on the challenges individuals with SZ can have when it comes to understanding the mental state of others, among them metacognitive treatments $[25,55]$. The clinician can use psychoeducation, psychotherapy sessions, and the therapeutic relationship itself to help individuals with SZ make sense not only of what they themselves experience, but what others experience, feel and think. The current study suggests that the inclusion, in psychotherapy, of discussions on how to perceive emotions from other people's body movements could be useful.

\section{Declarations of interest}

None.

\section{Acknowledgements}

We thank the participants for their time and effort as well as the clinical and research personnel at the NORMENT KG Jebsen Centre for Psychosis Research in Oslo, Norway.

\section{Funding sources}

This work was supported by The South East Norway Regional Health Authority (grant 2010007 and 2017069 to AV) and The Department of Psychology, University of Oslo, Norway (postdoctoral grant to AV). 


\section{References:}

[1] Pinkham AE. Social cognition in schizophrenia. J Clin Psychiatry 2014;75 Suppl2:14-9. doi:10.4088/JCP.13065su1.04

[2] Premack D, Woodruff G. Does the cimpanzee have a theory of mind? Behavior Brain Sci 1978;1:515-526.

[3] Green MF, Penn DL, Bentall R, Carpenter WT, Gaebel W, Gur RC, et al. Social cognition in schizophrenia: an NIMH workshop on definitions, assessment and research opportunities. Schizophr Bull 2008;34:1211-20.

[4] Savla GN, Vella L, Armstrong CC, Penn DL, Twamley EW. Deficits in domains of social cognition in schizophrenia: a meta-analysis of the empirical evidence. Schizophr Bull 2013;39:979-992. doi:10.1093/schbul/sbs080

[5] Schmidt SJ, Mueller DR, Roder V. Social cognition as a mediator variable between neurocognition and functional outcome in schizophrenia: empirical review and new results by structural equation modeling. Schizophr Bull 2011;37 Suppl2:S41-54. doi:10.1093/schbul/sbr079

[6] Fett AK, Viechtbauer W, Dominguez MD, Penn DL, van Os J, Krabbendam L.The relationship between neurocognition and social cognition with functional outcomes in schizophrenia: a meta-analysis. Neurosci Biobehav Rev 2011;35:573-88. doi:10.1016/j.neubiorev.2010.07.001

[7] Brizio A, Gabbatore I, Tirassa M, Bosco FM. "No more a child, not yet an adult": studying social cognition in adolescence. Front Psychol 2015;6:1011

[8] Ochsner KN. The social-emotional processing stream: five core constructs and their translational potential for schizophrenia and beyond. Biol Psychiatry 2008;64:48-61. doi:10.1016/j.biopsych.2008.04.024

[9] Shamay-Tsoory SG, Shur S, Barcai-Goodman L, Medlovich S, Harari H, Levkovitz Y. Dissociation of cognitive from affective components of theory of mind in schizophrenia. Psychiatry Res 2007;149:11-23. doi:10.1016/j.psychres.2005.10.018

[10] Abu-Akel A, Bailey AL. The possibility of different forms of theory of mind impairment in psychiatric and developmental disorders. Psychol Med 2000;30:735-738.

[11] Mancuso F, Horan WP, Kern RS, Green MF. Social cognition in psychosis: multidimensional structure, clinical correlates, and relationship with functional outcome. Schizophr Res 2011;125:143-51. doi:10.1016/j.schres.2010.11.007

[12] Mitchell RL, Phillips LH. The overlapping relationship between emotion perception and theory of mind. Neuropsychologia 2015;70:1-10.doi:10.1016/j.neuropsychologia.2015.02.018

[13] Mehta UM, Bhagyavathi HD, Thirthalli J, Kumar KJ, Gangadhar BN. Neurocognitive predictors of social cognition in remitted schizophrenia. Psychiatry Res 2014; 219 :268-74. doi:10.1016/j.psychres.2014.05.055 
[14] Bora E, Yucel M, Pantelis C. Theory of mind impairment in schizophrenia: meta-analysis. Schizophr Res 2009;109:1-9. doi:10.1016/j.schres.2008.12.020

[15] Fretland RA, Andersson S, Sundet K, Andreassen OA, Melle I Vaskinn A. Theory of mind in schizophrenia: error types and associations with symptoms. Schizophr Res 2015;162:42-6. doi:10.1016/j.schres.2015.01.024

[16] Pickup GJ. Relationship between theory of mind and executive function in schizophrenia: a systematic review. Psychopathology 2008;41:206-213.

[17] Parola A, Berardinelli L, Bosco FM. Cognitive abilities and theory of mind in explaining communicative-pragmatic disorders in patients with schizophrenia. Psychiatry Res 2018;260:144-151.

[18] Brothers L, Ring B. A neuroethological framework for the representation of minds. J Cogn Neurosci $1992 ; 4$ :107-18. doi:10.1162/jocn.1992.4.2.107

[19] Montag C, Dziobek I, Richter IS, Neuhaus K, Lehmann A, Sylla R et al. Different aspects of theory of mind in paranoid schizophrenia: evidence from a video-based assessment. Psychiatry Res $2011 ; 186$ :203-9. doi:10.1016/j.psychres.2010.09.006

[20] Brüne M . Emotion recognition, 'theory of mind,' and social behavior in schizophrenia. Psychiatry Res 2005;133:135-47. doi:10.1016/j.psychres.2004.10.007

[21] Fett AK, Maat A, GROUP Investigators. Social cognitive impairment and psychotic symptoms: what is the nature of their associations? Schizophr Bull 2013; 39:77-85.

[22] Bosco FM, Bono A, Bara BG. Recognition and repair of communicative failures: the interaction between theory of mind and cognitive complexity in schizophrenic patients. $\mathrm{J}$ Comm Disord 2012; 45:181-197.

[23] Ventura J, Wood RC, Hellemann GS. Symptom domains and neurocognitive functioning can help differentiate social cognitive procesesses in schizophrenia: a meta analysis. Schizophr Bull 2013;39:102-11.

[24] Andreou C, Kelm L, Bierbrodt J, Braun V, Lipp M, Yassari AH et al. Factors contributing to social cognition impairment in borderline personality disorder and schizophrenia. Psychiatry Res 2015;229:872-9.

[25] de Jong A, van Doenkersgoed RJM, Timmermann ME, aan het Rot M, Wunderink L, van Der Gaag M et al. Metacognitive reflection and insight therapy (MERIT) for patients with schizophrenia. Psychol Med 2018; Epub ahead of print doi: 10.1017/S0033291718000855

[26] Lysaker PH, Erickson M, Buck KD, Procacci M, Nicolò G, Dimaggio G. Metacognition in schizophrenia spectrum disorders: Methods of assessment and associations with neurocognition and function. Eur J Psychiatry 2010;4:220-226.

[27] Nicolò G, Dimaggio G, Popolo R, Carcione A, Procacci M, Hamm J et al. Associations of metacognition with symptoms, insight and neurocognition in clinically stable outpatients 
with schizophrenia. J Nerv Mental Dis, 2012;200:644-647.

[28] Vohs JL, Lysaker PH, Francis M, Hamm J, Buck KD, Olesek K et al. Metacognition, social cognition, and symptoms in patients in first episode and prolonged psychosis.

Schizophr Res 2014;153:54-59.

[29] Lysaker PH, Shea AM, Buck KD, Dimaggio G, Nicolò G, Procacci M et al. Metacognition as a mediator of the effects of impairments in neurocognition on social function in schizophrenia spectrum disorders. Acta Psych Scand 2010;122:405-413.

[30] Hamm JA, Renard SB, Fogley RL, Leonhardt BL, Dimaggio G, Buck KD et al. Metacognition and social cognition in schizophrenia: stability and relationship to concurrent and prospective symptom sssessments. J Clin Psychology 2012;68:1303-1312.

[31] Kurtz MM, Richardson CL. Social cognitive training for schizophrenia: a meta analytic investigation of controlled research. Schizophr Bull 2012;38:1092-104.

[32] Horan WP, Green MF. Treatment of social cognition in schizophrenia: current status and future directions. Schizophr Res 2017; doi: 10.1016/j.schres.2017.07.013.

[33] Dziobek I, Fleck S, Kalbe E, Rogers K, Hassenstab J, Brand M. Introducing MASC: a movie for the assessment of social cognition. J Autism Dev Disord 2006;36:623-36. doi:10.1007/s10803-006-0107-0

[34] Wechsler D.Wechsler Abbreviated Scale of Intelligence (WASI). Norwegian Manual Supplement. Stockholm, Sweden: Pearson Assessment; 2007.

[35] Spitzer RL, Williams JB, Kroenke K, Linzer M, deGruy FV $3^{\text {rd }}$, Hahn SR et al. Utility of a new procedure for diagnosing mental disorders in primary care. The PRIME-MD 1000 study. JAMA 1994;272:1749-56.

[36] Pedersen G, Hagtvedt KA, Karterud S. Generalizibility studies of the global assessment of functioning-split version. Compr Psychiatry 2007;48:88-94.

[37] American Psychiatric Association. The Diagnostic and Statistical Manual of Mental Disorders. 4th ed. Washington DC: American Psychiatric Publishing, 2000.

[38] First MB, Spitzer RL, Gibbon M, Williams JBW. Structured Clinical Interview for DSM-IV TR Axis I Disorders, Research Version, Patient Edition (SCID-P). New York State Psychiatric Institute, New York: Biometrics Research, 1996.

[39] Kay SR, Fiszbein A, Opler LA. The positive and negative syndrome scale (PANSS) for schizophrenia. Schizophr Bull 1987;13:261-276.

[40] Wallwork RS, Fortgang R, Hashimoto R, Weinberger DR, Dickinson D. Searching for a consensus five-factor model of the Positive and Negative Syndrome Scale for schizophrenia. Schizophr Res 2012;137:246-50. doi:10.1016/j.schres.2012.01.031

[41] Emsley R, Rabinowitz J, Torreman M. The factor structure for the Positive and Negative Syndrome Scale (PANSS) in recent-onset psychosis. Schizophr Res 2003;61:47-57. 
[42] Langeveld J, Andreassen OA, Auestad B, Faerden A, Hauge, LJ, Joa I et al. Is there an optimal factor structure of the Positive and Negative Syndrome Scale in patients with first episode psychosis? Scand J Psychol 2013;54:160-5. doi:10.1111/sjop.12017

[43] van der Gaag M, Hoffman T, Remijsen M, Hijman R, de Haan L, van Meijel B. et al. The five factor model of the Positive and Negative Syndrome Scale II: a ten-fold cross validation of revised model. Schizophr Res 2006;85:280-7.

doi:10.1016/j.schres.2006.03.021

[44] Montag C, Neuhaus K, Lehmann A, Kruger K, Dziobek I, Heekeren HH et al. Subtle deficits of cognitive theory of mind in unaffected first-degree relatives of schizophrenia patients. Eur Arch Psychiatry Clin Neurosci 2012;262:217-26.

[45] Newbury-Helps J, Feigenbaum J, Fonagy P. Offenders with antisocial personality disorder display more impairments in mentalizing. J Pers Disord 2016;31:232 -55. doi:10.1521/pedi_2016_30_246

[46] Heberlein AS, Adolphs R, Tranel D, Damasio H.Cortical regions for judgments of emotions and personality traits from point-light walkers. J Cogn Neurosci 2004;16:1143-58. doi:10.1162/0898929041920423

[47] Couture SM, Penn DL, Losh M, Adolphs R, Hurley R, Piven J. Comparison of social cognitive functioning in schizophrenia and high functioning autism: more convergence than divergence. Psychol Med 2010;40:569-79. doi:10.1017/s003329170999078x

[48] Vaskinn A, Sundet K, Østefjells T, Nymo K, Melle I, Ueland T. Reading emotions from body movement: a generalized impairment in schizophrenia. Front Psychol 2016;6:2058

[49] Nuechterlein KH, Green MF. MATRICS Consensus Cognitive Battery. Norwegian version. (Rund, B.R., Sundet, K. Eds). Los Angeles, CA : MATRICS Assessment, 2009.

[50] Crespi B, Badcock C. Psychosis and autism as diametrical disorders of the social brain. Behav Brain Sci 2008;31:241-61.

[51] Chisholm K, Lin A, Abu-Akel A,Wood SJ. The association between autism and schizophrenia spectrum disorders: a review of eigth alternate models of co-occurrence. Neurosci Biobeh Rev 2015;55:173-83.

[52] Kincaid DL, Doris M, Shannon C, Mulholland C. What is the prevalence of autism spectrum disorder and ASD traits in psychosis? A systematic review. Psychiatry Res 2017; 250:99-105.

[53] Kahn RS, Keefe RS. Schizophrenia is a cognitive illness: time for a change in focus. JAMA Psychiatry 2013;70:1107-12.

[54] Van Rheenen TE, Rossell SL. An empirical evaluation of the MATRICS Consensus Cognitive Battery in bipolar disorder. Bipolar Disord 2014;16:318-325.

doi:10.1111/bdi.12134 
[55] Inchausti F, García-Poveda NV, Ballesteros-Prados A, Ortuño-Sierra J, Sánchez-Reales $\mathrm{S}$, Prado-Abril J et al. The effects of metacognition-oriented social skills training on psychosocial outcome in schizophrenia-spectrum disorders: a randomized controlled trial. Schizophr Bull 2017; Epub ahead of print doi: 10.1093/schbul/sbx168 


\section{Appendix.}

Examples of MASC response categories.

While the four characters prepare dinner together, one of the male characters insults, tonguein-cheek, one of the female characters by saying that if it were up to her, they would have added five instead of two cups of cream to the sauce.

Q: What does she feel?

Response options:

A1. Insulted by his comment (correct).

A2. Hates him and wants him to leave (incorrect: overmentalizing).

A3. Amazed that he knows that she likes cream (incorrect: undermentalizing).

A4. Five cups is too much for this sauce (incorrect: no mentalizing). 